\title{
Radiation Bursts from a Presupernova Collapsar
}

\author{
Volodymyr Kryvdyk \\ Kyiv National University, av. Glushkova 6, Kyiv 03022, Ukraine; \\ kryvdyk@mail.univ.kiev.ua
}

\begin{abstract}
Summary. The radiation from the magnetic presupernova star is calculated. This radiation will generate when the magnetosphere of presupernova star compresses during collapse and its magnetic field increases considerably. The variable magnetic field will accelerate the charged particle, which generate radiation when moving in the magnetic field. The particles dynamics and their non-thermal emission in the magnetospheres of presupernova collapsing star with initial dipole magnetic fields and a certain initial energy distribution of charged particles in a magnetosphere are considered. The radiation flux depend on the distance to the star, its magnetic field, and the particle spectrum in the magnetosphere. This flux can be observed by means of modern instruments in broad band (from radio waves to gamma rays). The radiation flux grows with decreasing stellar radius and frequency and can be observed in the form of radiation bursts with duration equal to the stellar collapse time.
\end{abstract}

\section{Introduction}

Collapsing stars have investigated first Chandrasekhar and OppenheimerVolkoff in papers [1, 13]. Collapse begins when the mass of stellar core exceeds the Chandrasekhar Limit, and the star become dynamically unstable. The star compress and its radius decrease. The stellar magnetic field grows during the collapse [2]. The stars must emit electromagnetic and the gravitational waves under the collapse $[3,4,5,6,11]$. Today we can not detected these waves near Earth over the very low frequencies these waves $[3,4,5,11]$, and maybe it is the main problem in the observation of stellar collapse. Therefore the collapsing stars are so far only the theoretical objects and not detected directly by astronomical observations. Of course, we can detected such phenomenon as the explosion of supernova stars and their emission $[10,12]$. But the explosion takes place after collapse and this phenomenon is a consequence of the gravitational collapse.

In our paper we propose a test for search of the presupernova stars on the stage of gravitational collapse using the non-thermal emission from the magnetospheres of presupernova collapsing stars. 


\section{Presupernova Collapsar Magnetosphere}

When the star magnetosphere compresses under the collapse, its magnetic field considerably increases. The cyclic electric field thus produced, accelerate charged particles that generate radiation when moving in the magnetic field. The field structure and particles dynamics in the magnetosphere are influenced by three factors: particles pressure, collisions, and star rotation. As follow with the detail analysis [7], these effects can be neglected during the collapse. To investigate particle dynamics in magnetosphere the method of adiabatic invariant have used as since the magnetospheric plasmas is frozen in magnetic field and collision-free. In this case there are two mechanisms of the particle interaction with the magnetic fields. First is betatron acceleration in the variable magnetic field, second is bremsstrahlung energy losses in this field.

The external electromagnetic field of a collapsing star is given by [7] $B(r, \theta, t)=(1 / 2) F_{o} R r^{-3}\left(1+3 \cos ^{2} \theta\right)^{1 / 2}$. Here $F_{o}=B_{o} R_{o}^{2}$ is the initial magnetic flux of star with the radius $R$ having the initial radius $R_{o}$ and the initial magnetic field $B_{o}$.

The particles spectrum for such magnetosphere [7] is $N_{p}(E, R)=$ $K_{p} E^{-\gamma} R_{*}^{-\beta_{1}} N_{M}(E, R)=K_{M} E^{2} R_{*}^{-\beta_{2}} \exp (-E / k T), N_{B}(E, R)=K_{B} R_{*}^{-\beta_{3}}$ $\exp (-E / k T)$, respectively for the initial a power -law, relativistic Maxwell and Boltzmann distributions of charged particles in the magnetosphere. Here $K_{C}, K_{M}, K_{B}$ are the spectral coefficients; $k$ is the Boltzmann constant; $E$ is the particle energy and $T$ is the temperature in the magnetosphere; $\gamma$ is the power spectrum, $R_{*}=R_{0} / R, \quad \beta_{1}=a_{1}(\gamma-1) ; \quad \beta_{2}=a_{1}(E / k T \ln E-3)$; $\beta_{3}=a_{1}(E / k T \ln E-1), a_{1}=\left(5 k_{1} / 3\right)\left(3 \cos ^{4} \theta+1.2 \cos ^{2} \theta-1\right)\left(1+3 \cos ^{2} \theta\right)^{-2}$; $k_{1}=1$ and $k_{1}=2$ for relativistic and non-relativistic particles, respectively.

\section{Non-thermal Radiation from Presupernova Collapsar}

The ratio between the radiation flux from the presupernova collapsing stars on the any stage of collapse (when the stellar radius decrease to the value $R$ ) and its initial radiation flux (when the radius is $R_{0}$ ), respectively for the power-law, relativistic Maxwell, and Boltzmann distributions are [8]

$$
\begin{gathered}
I_{\nu P} / I_{\nu P 0}=\left(\nu / \nu_{0}\right)^{(1-\gamma) / 2} R_{*}^{\gamma-2}\left(I_{\nu P 2} / I_{\nu P 20}\right) \\
I_{\nu M} / I_{\nu M 0}=R_{*}^{-3}\left(\nu / \nu_{0}\right)(1 / k T) \int_{0}^{\infty} \int_{0}^{\pi / 2} R_{*}^{-\beta_{2}} \exp (-E / k T) \sin \theta d E d \theta
\end{gathered}
$$




$$
I_{\nu B} / I_{\nu B 0}=R_{*}^{-3}(k T)\left(\nu / \nu_{0}\right) \int_{0}^{\infty} \int_{0}^{\pi / 2} R_{*}^{-\beta_{3}} E^{-2} \exp (-E / k T) \sin \theta d E d \theta
$$

Using equations (1)-(3) the radiation flux from presupernova collapsar can be calculated. The ratio between the radiation flux from collapsing stars and its initial flux by $\nu / \nu_{0}=1$ are in the ranges:

$$
\begin{array}{rlrl} 
& 1 \leq I_{\nu P} / I_{\nu P 0} \leq 1.34 \times 10^{10} & & \text { for } 2.4 \leq \gamma \leq 3.4 \\
\leq & R_{*} \leq 1000 ; & \\
& 1 \leq J_{\nu M} / J_{\nu M 0} \leq 4.86 \times 10^{5} & & \text { for } 1 \mathrm{eV} \leq k T \leq 9 \mathrm{eV} \\
R_{*} & \leq 850 ; & & \\
& 1 \leq J_{\nu B} / J_{\nu B 0} \leq 2.23 \times 10^{11} & & \text { for } 1 \mathrm{eV} \leq k T \leq 9 \mathrm{eV},
\end{array}
$$

$R_{*} \leq 850$ $R_{*} \leq 850$

These values obtained by the numerical integration of the equations for the ratio between the radiation flux in the range $2 \mathrm{eV} \leq E \leq 10^{9} \mathrm{eV}, 0 \leq$ $\theta \leq \pi / 2$ for the different radius $R_{*}$, temperature $k T$ and index $\gamma$.

\section{Conclusions}

We can see that the presupernova stars on the stage of gravitational collapse can be the powerful sources of the non-thermal radiation impulses. Then collapsing star can lose the part their mass and flare up as supernova with a expanding shell. But before the explosion supernova star must precede the stage of gravitational collapse when the star compress under the influence of gravitation. On this stage a star is the powerful source of the non-thermal radiation. The impulse of the non-thermal radiation can observed also before explosion of nova. The powerful sources of the non-thermal radiation can be also the white dwarfs in double systems on the stage accretion induced collapse [9]. Periodic impulses of non-thermal radiation can be generated also by pulsation of the stars with magnetic field, since in this case the charged particles can accelerate and the non-thermal emission generate.

What problems can arise by the astrophysical observation of the nonthermal radiation from the collapsing presupernova stars? First the theory of the stellar evolution does not enable us today to point to the location of presupernova stars before their explosion. This fact is principal problem in the astrophysical observation for the search of presupernova collapsing stars. Second problems is that the stellar collapse pass very rapidly. But this problem can be solved since the modern instruments enable to observe the very short impulse. Next problems is how to choose the impulse from presupernova collapsing stars from the great numbers of bursts with the unknown origin. These questions require additional investigations.

\section{References}

1. S. Chandrasekhar: Mon. Not. R. Astron. Soc. 95, 207 (1935) 
2. V.L. Ginzburg, L.M. Ozernoy: J. Theor. \& Experiment. Phys. 47, 1030 (1964)

3. C.T. Gunningham, R.H. Price, V. Moncrief: Astrophys. J. 224, 643 (1978)

4. C.T. Gunningham, R.H. Price, V. Moncrief: Astrophys. J. 230, 870 (1979)

5. C.T. Gunningham, R.H. Price, V. Moncrief: Astrophys. J. 236, 674 (1980)

6. R.N. Henriksen, W.Y. Chau, K.L.Chau: Astrophys. J. 227, 1013 (1979)

7. V. Kryvdyk: Mon. Not. R. Astron. Soc. 309, 593 (1999)

8. V. Kryvdyk: Adv. Space Res. 28, 463 (2001)

9. V. Kryvdyk: NATO ASIB Proc. 10, 205 (2003)

10. A.I. MacFadyen, S.E. Woosley: Astrophys. J. 524, 262 (1999)

11. V. Moncrief: Astrophys. J. 238, 333 (1980)

12. M. Milgrom, V.V. Usov: Astrophys. J. Lett. 531, L127 (2000)

13. I.R. Oppenheimer, G. Volkoff : Phys. Rev. 55, 374 (1939) 